\title{
Double polarization experiments at intermediate energy
}

\author{
H. Schmieden ${ }^{\mathrm{a}}$

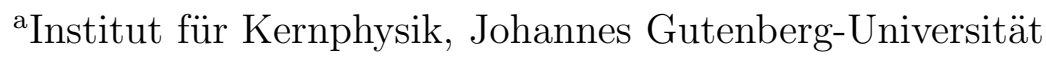 \\ J.J. Becher-Weg 45, 55099 Mainz, Germany
}

At modern electron accelerators with highly polarized, intense, high duty factor beams double polarization coincidence experiments became feasible with good statistical accuracy. The strong potential towards the precise determination of small nucleon structure quantities is illustrated by two recent examples from MAMI. The measurement of $G_{E}^{n}$ in the quasifree reaction $D\left(\vec{e}, e^{\prime} \vec{n}\right) p$ lead to a new parametrization of $G_{E}^{n}$ which is significantly above the previously preferred one from elastic $e-D$ scattering. A $p\left(\vec{e}, e^{\prime} \vec{p}\right) \pi^{0}$ experiment at the energy of the $\Delta$ resonance yields preliminary results for the longitudinal quadrupole mixing. Both experimental errors and model uncertainties are complementary to unpolarized measurements.

\section{INTRODUCTION}

Polarization experiments offer the possibility to measure interferences of different amplitudes. This is particularly interesting in the situation of a small quantity in the vicinity of a dominating large one. Due to the insensitivity of polarization observables to many calibration factors the small quantities can be reliably extracted.

For these reasons a variety of experimental programs in the intermediate energy range has been established at the electron accelerator facilities ELSA, MAMI, MIT-Bates, NIKHEF and TJNAF. In the following, two recent examples of double polarization experiments at MAMI will be discussed: the measurement of the neutron electric form factor, $G_{E}^{n}$, in the quasifree $D\left(\vec{e}, e^{\prime} \vec{n}\right) p$ reaction, and the extraction of the longitudinal quadrupole mixing in the $\mathrm{N}$ to $\Delta$ transition from $p\left(\vec{e}, e^{\prime} \vec{p}\right) \pi^{0}$.

Both experiments require longitudinally polarized, high duty factor beams in combination with recoil polarimetry. The nucleons are detected in parallel kinematics, i.e. along the direction of the momentum transfer, $\vec{q}$. The cartesian components of the nucleon polarization, $P_{x, y, z}$, are advantageously expressed in the plane of incoming $\left(\vec{k}_{i}\right)$ and scattered electron $\left(\vec{k}_{f}\right): \hat{y}=\left(\vec{k}_{i} \times \vec{k}_{f}\right) /\left|\vec{k}_{i} \times \vec{k}_{f}\right|, \hat{z}=\vec{q} /|\vec{q}|$, and $\hat{x}=\hat{y} \times \hat{z}$.

\section{MEASUREMENT OF $G_{E}^{n}$ via $D\left(\vec{e}, e^{\prime} \vec{n}\right) p$}

Double polarization observables in quasifree electron-deuteron scattering offer high sensitivity to $G_{E}^{n}$ due to an interference with the large magnetic form factor, $G_{M}^{n}$, combined with negligible dependence on the deuteron wavefunction [1]. For the ideal case of free electron-neutron scattering, $n\left(\vec{e}, e^{\prime} \vec{n}\right)$, Arnold, Carlson and Gross [2] obtained for the 


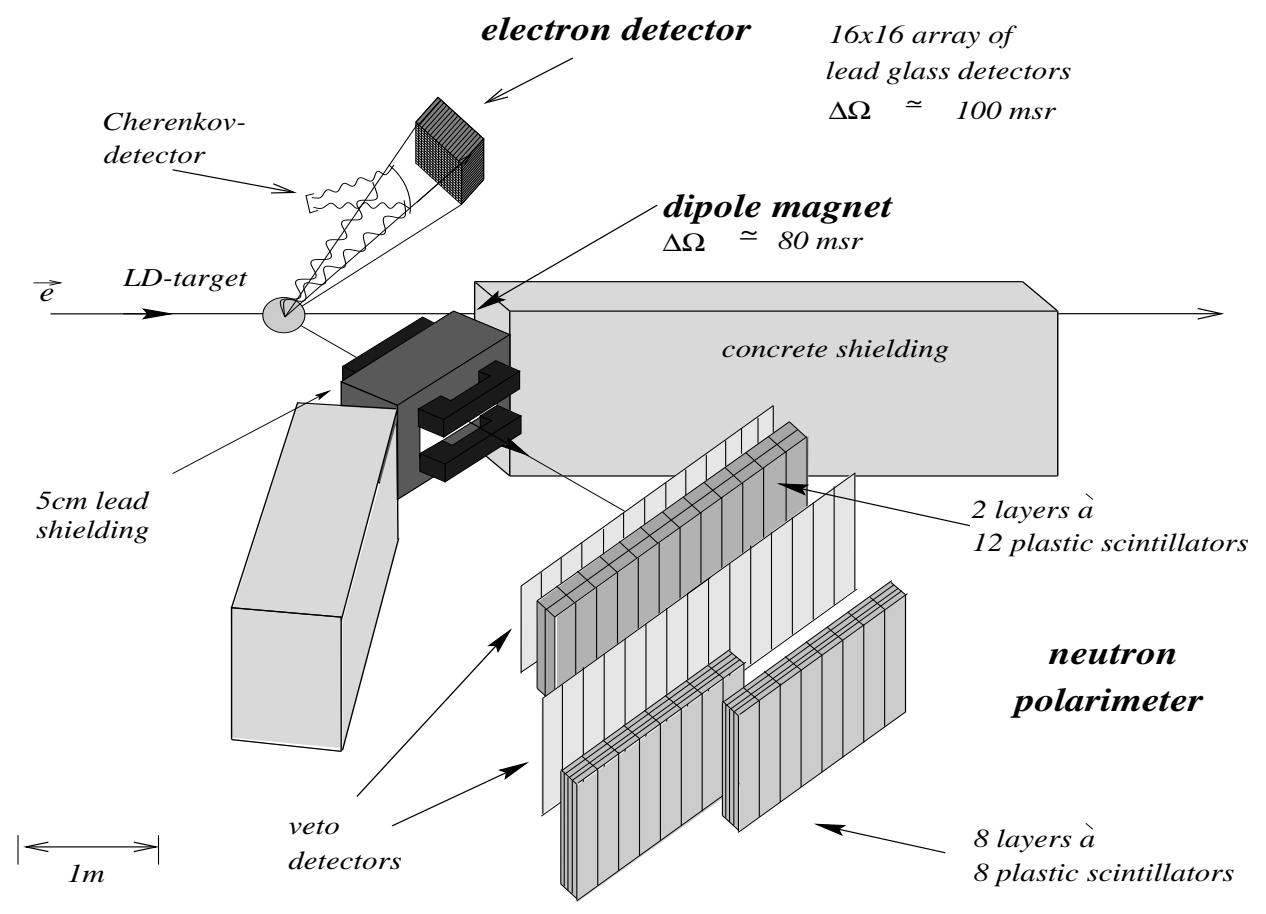

Figure 1. Setup of the $D\left(\vec{e}, e^{\prime} \vec{n}\right)$ experiment at MAMI

components of the recoil polarization'

$$
\begin{aligned}
P_{x} & =-P_{e} \frac{\sqrt{2 \tau \epsilon(1-\epsilon)} G_{E}^{n} G_{M}^{n}}{\epsilon\left(G_{E}^{n}\right)^{2}+\tau\left(G_{M}^{n}\right)^{2}} \\
P_{y} & =0 \\
P_{z} & =P_{e} \frac{\tau \sqrt{1-\epsilon^{2}}\left(G_{M}^{n}\right)^{2}}{\epsilon\left(G_{E}^{n}\right)^{2}+\tau\left(G_{M}^{n}\right)^{2}} .
\end{aligned}
$$

$\epsilon=\left(1+\frac{2|\vec{q}|^{2}}{Q^{2}} \tan ^{2} \frac{\vartheta_{e}}{2}\right)^{-1}$ is the photon polarization parameter, $Q^{2}=-q_{\mu} q^{\mu}$ the squared four momentum transfer and $\tau=Q^{2} / 4 m_{n}^{2}$ represents the momentum transfer in units of the neutron mass, $m_{n} . \vartheta_{e}$ denotes the electron scattering angle, and $P_{e}$ the longitudinal polarization of the electron beam.

The longitudinally polarized electron beam $\left(I \simeq 2.5 \mu \mathrm{A}, P_{e} \simeq 75 \%\right)$ hit a $5 \mathrm{~cm}$ long liquid deuterium target and the scattered electrons were detected in a 256 element lead glass array (Fig.11). The energy resolution of $\delta E / E \simeq 25 \%$ was sufficient to suppress pion production events. Only the electron angles, which were measured with an accuracy of $\delta \vartheta, \delta \phi \simeq 3.5 \mathrm{mrad}$ entered the event reconstruction, which became kinematically complete through the measurement of the neutrons time-of-flight and hit position in the front plane of the neutron detector.

\footnotetext{
${ }^{1}$ Equivalently, the scattering of longitudinally polarized electrons off a polarized neutron target leads to a cross section asymmetry with regard to reversal of beam helicity.
} 

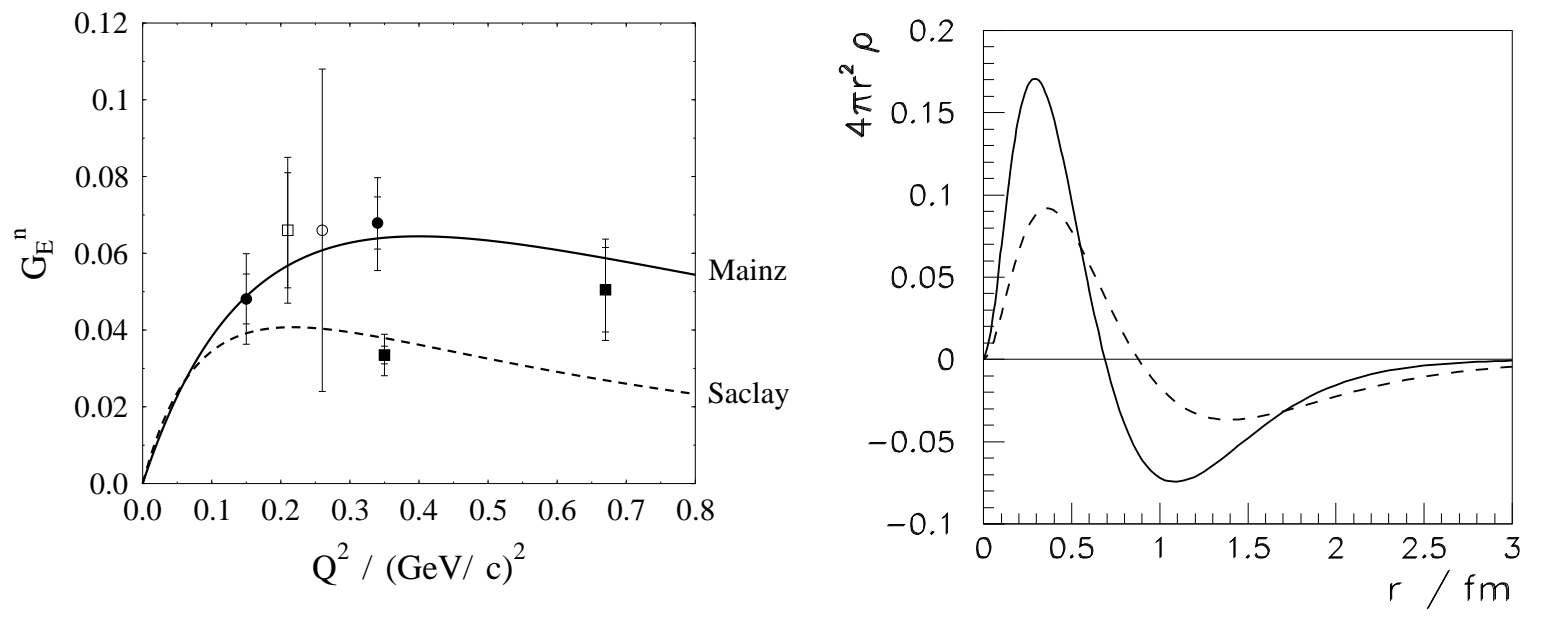

Figure 2. Results for $G_{E}^{n}$ from double polarization experiments. The data points and curves are discussed in the text.
Figure 3. Neutron charge distribution for the Mainz (full) and the preferred Saclay (broken) $G_{E}^{n}$ parametrization (see text).

The neutron polarization can be analyzed in the detection process itself [3]. This required a second neutron detection in one of the rear detector planes, which yielded the polar and azimuthal angles, $\Theta_{n}^{\prime}$ and $\Phi_{n}^{\prime}$, of the analyzing scattering in the front wall. With the number of events $N^{ \pm}\left(\Phi_{n}^{\prime}\right)$ for \pm helicity states of the electron beam the azimuthal asymmetry, $A\left(\Phi_{n}^{\prime}\right)$, was determined through the ratio

$$
\frac{1-A\left(\Phi_{n}^{\prime}\right)}{1+A\left(\Phi_{n}^{\prime}\right)}=\sqrt{\frac{N^{+}\left(\Phi_{n}^{\prime}\right) \cdot N^{-}\left(\Phi_{n}^{\prime}+\pi\right)}{N^{-}\left(\Phi_{n}^{\prime}\right) \cdot N^{+}\left(\Phi_{n}^{\prime}+\pi\right)}},
$$

which is insensitive to detector efficiencies and luminosity variations. The extraction of $P_{x}$ from $A\left(\Phi_{n}^{\prime}\right)=\epsilon_{\mathrm{eff}} \cdot P_{x} \cdot \sin \Phi_{n}^{\prime}$ requires the calibration of the effective analyzing power, $\epsilon_{\text {eff, }}$ of the polarimeter. This, however, varies strongly with the event composition as determined by hardware conditions during data taking and software cuts applied in the offline analysis.

For the first time, the problem of calibration of the effective analyzing power has been avoided by controlled precession of the neutron spins in the field of a dipole magnet in front of the polarimeter [4]. After precession by the angle $\chi$ the transverse neutron polarization behind the magnet, $P_{\perp}$, is a superposition of $x$ and $z$ components, and likewise is the measured asymmetry:

$$
A_{\perp}=A_{x} \cos \chi-A_{z} \sin \chi .
$$

For the particular case of the zero crossing, $A_{\perp}\left(\chi_{0}\right)=0$, one immediately gets the relation

$$
\tan \chi_{0}=\frac{A_{x}}{A_{z}}=\frac{\epsilon_{\mathrm{eff}} \cdot P_{e} \cdot \sqrt{2 \tau \epsilon(1-\epsilon)} G_{E}^{n} \cdot G_{M}^{n}}{\epsilon_{\mathrm{eff}} \cdot P_{e} \cdot \tau \sqrt{1-\epsilon^{2}}\left(G_{M}^{n}\right)^{2}} .
$$


Obviously, this ratio is independent of both $P_{e}$ and $\epsilon_{\text {eff }}$ and it directly yields $G_{E}^{n} / G_{M}^{n}$. The neutron electric form factor has been extracted relying on the dipole values for $G_{M}^{n}$.

Data have been taken around $Q^{2}=0.32(\mathrm{GeV} / \mathrm{c})^{2}$ and $Q^{2}=0.12(\mathrm{GeV} / \mathrm{c})^{2}$. According to calculations of $\mathrm{H}$. Arenhövel the effect of final state interaction on $P_{x}-$ which is dominated by charge exchange of the outgoing nucleons - is almost negligible at the higher momentum transfer. However, due to the small relative energy in the n-p final state, it becomes important at the small $Q^{2}$. For the MAMI $D\left(\vec{e}, e^{\prime} \vec{n}\right)$ data the influence of FSI has been explicitly studied for the first time [5]. A $100 \%$ correction is required at $Q^{2}=0.12(\mathrm{GeV} / \mathrm{c})^{2}$ which drops to $8 \%$ at $Q^{2}=0.35(\mathrm{GeV} / \mathrm{c})^{2}$. The corrected results are depicted in Fig.2 as full circles with statistical (inner) and systematical (outer) error. A FSI correction is implicitly also included in the NIKHEF result [6] (open square), but not in the Bates one [0] (open circle). The full squares represent the uncorrected MAMI results for ${ }^{3} \overrightarrow{H e}\left(\vec{e}, e^{\prime} n\right)$. At $Q^{2}=0.6(\mathrm{GeV} / \mathrm{c})^{2}$ [8 FSI is expected to be negligible due to the large kinetic energy of the ejected neutron. However, first, still incomplete 3-body calculations indicate a substantial correction of the data point at $Q^{2}=0.36(\mathrm{GeV} / \mathrm{c})^{2}($ full square) [9] towards larger $G_{E}^{n}$. Excluding this data point, a new fit of the dipole-ansatz

$$
G_{E}^{n}=-\frac{\mu_{n} \tau}{1+\eta \tau} \cdot\left[1+\frac{Q^{2}}{0.71(G e V / c)^{2}}\right]^{-2}
$$

to the recent MAMI double polarization results yielded $\eta=3.4 . \mu_{n}$ is the neutron magnetic moment. As shown in Fig.2, this fit lies almost a factor of two above the previously favoured result from elastic $D\left(e, e^{\prime}\right)$ scattering, where the Paris potential has been used for the unfolding of the wave function contribution [10]. This causes a significant difference of the neutron charge distributions as obtained by Fourier transformation of $G_{E}^{n}$. The full and broken curve in Fig. 3 correspond to the Mainz and Saclay $G_{E}^{n}$ parametrizations, respectively.

\section{RECOIL POLARIZATION IN THE $p\left(\vec{e}, e^{\prime} \vec{p}\right) \pi^{0}$ REACTION AND THE C2/M1 RATIO IN THE N TO $\Delta$ TRANSITION}

The spherical symmetry of the distributions of charge and magnetism within the nucleon was discussed since the early days of the quark model [11], although the nucleon's spin $1 / 2$ forbids the existence of a static quadrupole moment. However, in the $N \rightarrow \Delta_{33}(1232)$ transition quadrupole components are allowed aside of the dominant spin-flip of the unaligned constituent quark. In the almost exclusive decay of the $\Delta_{33}(1232)$ resonance into the $N \pi$ channel, this small quadrupole mixing is associated with small, but non-zero, electric quadrupole to magnetic dipole (EMR) and Coulomb quadrupole to magnetic dipole ratios (CMR). These are defined as:

$$
\begin{aligned}
& \mathrm{EMR}=\Im m\left\{E_{1+}^{3 / 2}\right\} / \Im m\left\{M_{1+}^{3 / 2}\right\} \\
& \mathrm{CMR}=\Im m\left\{S_{1+}^{3 / 2}\right\} / \Im m\left\{M_{1+}^{3 / 2}\right\},
\end{aligned}
$$

where the pion multipoles, $A_{l_{\pi}}^{I}$, are characterized through their magnetic, electric or longitudinal (scalar) nature, $A$, the isospin, $I$, and the pion-nucleon relative angular momentum, $l_{\pi}$, whose coupling with the nucleon spin is indicated by \pm . 
While the EMR has recently been measured at the photon point $\left(Q^{2}=0\right)$ 12, 13, the determination of the longitudinal quadrupole mixing requires pion electroproduction experiments. Double polarization observables in the $p\left(\vec{e}, e^{\prime} \vec{p}\right) \pi^{0}$ reaction offer high sensitivity to the CMR. In parallel kinematics, the components of the proton polarization are given in $\mathrm{s}^{-}$and p-wave approximation by [14] 2]:

$$
\begin{aligned}
\sigma_{0} P_{x}= & P_{e} \cdot c_{-} \cdot \Re e\left\{\left(4 S_{1+}+S_{1-}-S_{0+}\right)^{*} \cdot\left(M_{1+}-M_{1-}-E_{0+}+3 E_{1+}\right)\right\} \\
\sigma_{0} P_{y}= & c_{+} \cdot \Im m\left\{\left(4 S_{1+}+S_{1-}-S_{0+}\right)^{*} \cdot\left(M_{1+}-M_{1-}-E_{0+}+3 E_{1+}\right)\right\} \\
\sigma_{0} P_{z}= & P_{e} \cdot \sqrt{1-\epsilon^{2}} \cdot\left(\left|M_{1+}\right|^{2}+\left|M_{1-}\right|^{2}+9\left|E_{1+}\right|^{2}+\left|E_{0+}\right|^{2}+\right. \\
& \left.+\Re e\left\{6 E_{1+}^{*}\left(M_{1+}-M_{1-}\right)-2 M_{1+}^{*} M_{1-}-2 E_{0+}^{*}\left(M_{1+}-M_{1-}+3 E_{1+}\right)\right\}\right), 1
\end{aligned}
$$

where $c_{ \pm}=\sqrt{2 \epsilon_{L}(1 \pm \epsilon)} \frac{\omega_{c m}}{\left|\vec{q}_{c m}\right|} \cdot \omega_{c m}$ and $\vec{q}_{c m}$ denote the energy and momentum transfer in the cm-frame and $\epsilon_{L}=\frac{Q^{2}}{\omega_{c m}^{2}} \epsilon$. The $p-\pi^{0}$ multipoles of Eqs.10- 12 , $A_{l \pm}$, are related to the isospin multipoles by $A_{l \pm}=A_{l \pm}^{1 / 2}+\frac{2}{3} A_{l \pm}^{3 / 2}$.

As a consequence of the $M_{1+}^{3 / 2}$ dominance, $\Re e M_{1+}$ vanishes very closely to the resonance position $(W=1232 \mathrm{MeV})$, i.e.

$$
\begin{aligned}
& \sigma_{0} P_{x}=P_{e} \cdot c_{-} \cdot 4 \Im m S_{1+} \Im m M_{1+}+\text { n.l.o. } \\
& \sigma_{0} P_{y}=c_{+} \cdot 4 \Re e S_{1+} \Im m M_{1+}+\text { n.l.o. } \\
& \sigma_{0} P_{z}=P_{e} \cdot \sqrt{1-\epsilon^{2}}\left|M_{1+}\right|^{2}+\text { n.l.o. }
\end{aligned}
$$

$S_{1+}, E_{1+}$ and $M_{1+}$ are the only multipoles which couple to the $\Delta$ resonance, i.e. possess significant imaginary parts. With a purly Born - i.e. real - background the imaginary parts of the other multipoles vanish. Rescattering effects produce only small imaginary parts in the $S_{0+}$ and the $E_{0+}$ amplitudes. Therefore the higher order contributions, n.l.o., to $P_{x}$ (Eq.13) can expected to be small. The situation is different for $P_{y}$, because here all the real Born multipoles contribute (Eq.11). From Eqs.13 and 15 it is evident that the CMR can be almost directly determined from the polarization ratio $R=\frac{\sqrt{1-\epsilon^{2}}}{4 c_{-}} \frac{P_{x}}{P_{z}}$ 《14.

At the 3-spectrometer setup [17] of the Mainz microtron MAMI a $p\left(\vec{e}, e^{\prime} \vec{p}\right) \pi^{0}$ experiment with longitudinally polarized electron beam and measurement of the recoil proton polarization has been performed. At $Q^{2}=0.121(\mathrm{GeV} / \mathrm{c})^{2}$ a range of invariant energies of $W=1200 \ldots 1260 \mathrm{MeV}$ was covered.

The proton polarization was measured through the standard technique of inclusive $\mathrm{p}$ ${ }^{12} \mathrm{C}$ scattering. Therefore the detector package of spectrometer A was supplemented by a $7 \mathrm{~cm}$ thick carbon scatterer followed by two double planes of horizontal drift chambers [18. This setup allowed the eventwise reconstruction of the proton carbon scattering angles $\Theta_{C}$ and $\Phi_{C}$ with a resolution of $3 \mathrm{mrad}$. The azimuthal modulation of the cross section

$$
\sigma_{C}=\sigma_{C, 0}\left[1+A_{C}\left(P_{y}^{f p} \cos \Phi_{C}-P_{x}^{f p} \sin \Phi_{C}\right)\right]
$$

is related to the proton polarization; $\sigma_{C, 0}$ denotes the polarization independent part of the inclusive cross section and $A_{C}$ the analyzing power, which was parameterized according to [19].

\footnotetext{
${ }^{2}$ In contrast to ref. [14] here the conventions of ref. [15] are used. The different p-wave signs compared to
} 14 are due to an inconsistency in [16]. 

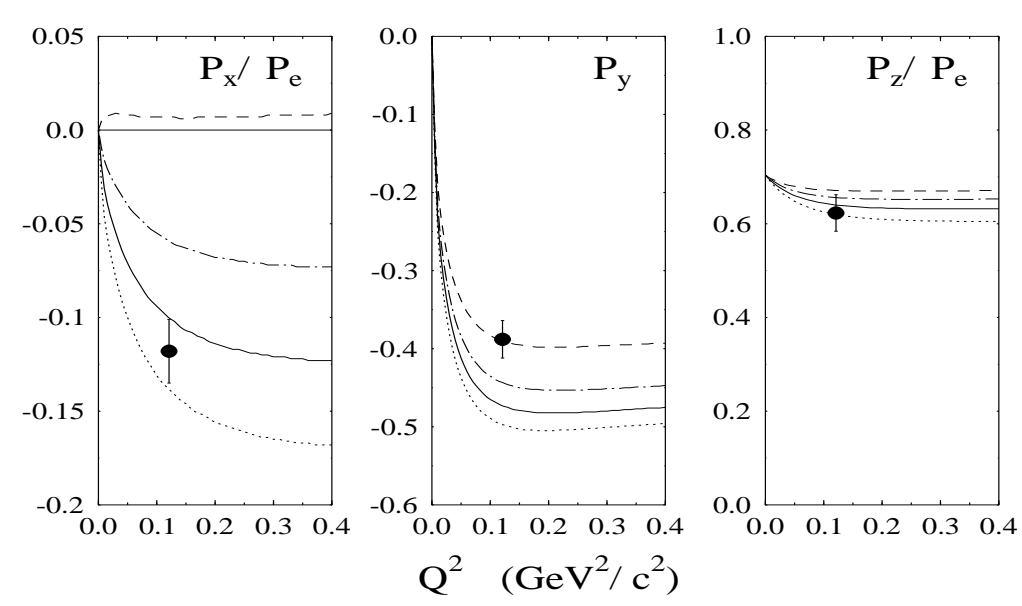

Figure 4. Preliminary results for the measured polarization components. The dashed, dot-dashed, full and dotted curves correspond to calculations within the UIM for $\mathrm{CMR}=0$, $-2.9,-4.8,-6.7 \%$, respectively.

The two polarization components $P_{x}^{f p}$ and $P_{y}^{f p}$ are measured behind the spectrometer's focal plane. It is possible to determine all three components at the electron scattering vertex due to the spin precession in the spectrometer and the redundancy provided by the helicity flip of the electron beam.

The spin precession along the proton trajectories through the magnetic fields of the QSDD spectrometer A [17] was computed by stepwise numerical integration of the BMTequation. On the basis of several thousand rays which were distributed over the large spectrometer acceptance a 5-dimensional spin precession matrix describing the spectrometer's 'polarization-optics' was generated [18]. The results were checked through the elastic scattering reaction $p\left(\vec{e}, e^{\prime} \vec{p}\right)$ where the proton polarization is determined by electron kinematics and the proton elastic form factors.

The matrix was then used for the extraction of the recoil proton polarization in the $p\left(\vec{e}, e^{\prime} \vec{p}\right) \pi^{0}$ reaction. The preliminary results for the three polarization components are shown in Fig. $\uplus$. The curves represent full (i.e. without restriction to s- and p-waves) calculations in the framework of the Mainz unitary isobar model (UIM) [20]. $P_{x}$ is very sensitive to the CMR and from this polarization component a value of CMR $=(-5.19 \pm$ $\left.0.75_{\text {stat }} \pm 0.60_{\text {syst }}\right) \%$ is extracted. However, since the influence of systematic uncertainties is reduced, it is preferrable to extract the CMR from the ratio $R=(-6.17 \pm 0.99 \pm 0.56) \%$. The preliminary result for the full UIM analysis of the ratio yields CMR $=(-5.25 \pm 0.82 \pm$ $0.48) \%$.

Although the CMR is determined in the $\pi^{0}$ channel, at the resonance position (defined by $\Re e M_{1+}^{3 / 2}=0$, i.e. at $W=1232 \mathrm{MeV}$ ) it is believed to come very close to the value for the isospin-3/2 channel (Eq.9) due to resonance dominance. In the UIM the 


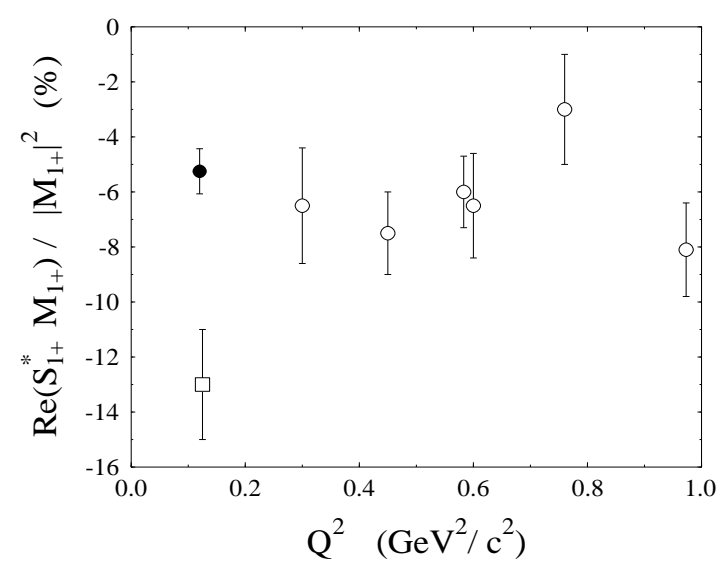

Figure 5. Preliminary result of the CMR as extracted from $P_{x} / P_{z}$ of this experiment (full circle) in comparison with unpolarized measurements from DESY, NINA, the Bonn synchrotron 21] (open circles), and ELSA [24] (open square). Errors are purely statistical.

$P_{x^{-}}$-interference in the $\pi^{0}$ channel practically equals at resonance the isospin-3/2 one: $\Re e\left\{S_{1+}^{\pi^{0} *} M_{1+}^{\pi^{0}}\right\} /\left|M_{1+}^{\pi^{0}}\right|^{2}=\Re e\left\{S_{1+}^{3 / 2 *} M_{1+}^{3 / 2}\right\} /\left|M_{1+}^{3 / 2}\right|^{2}$.

In Fig.5 the preliminary result is plotted along with published results from coincident $\pi^{0}$ experiments with unpolarized electrons. It is compatible with these data [21] and with recent, yet unpublished results [22,23], which are not included in Fig.5. However, one Bonn result [24] seems to be incompatible with all other data. Wether this is an experimental or statistical artefact, or a severe hint that there is another than expected $\Im m S_{0+}$ background contribution - which enters with different sign in the kinematics of that experiment compared to all other ones - is still an open problem but hoped to be decided soon 25].

\section{SUMMARY}

Two examples of double polarization experiments from MAMI in the medium energy range have been presented: quasifree $D\left(\vec{e}, e^{\prime} \vec{n}\right) p$ scattering and $p\left(\vec{e}, e^{\prime} \vec{p}\right) \pi^{0}$ in the energy range of the $\Delta$ resonance. These experiments resemble each other in that the recoiling nucleons are detected in coincidence with the scattered electrons in the direction of the momentum transfer and the nucleon polarization is analyzed in appropriate polarimeters. In both cases the interesting observables can be extracted from ratios of polarizations with the advantage of a particular insensitivity to calibration factors.

The new double polarization experiments in quasifree kinematics establish that the neutron electric form factor, $G_{E}^{n}$, is almost a factor of two higher than the previously favoured result of elastic $e-D$ scattering. This corresponds to a significant different charge distribution inside the neutron. 
The recoil polarization observables in the pion electroproduction reaction are sensitive to the longitudinal quadrupole mixing, CMR, in the $p \rightarrow \Delta^{+}$transition. The preliminary result seems to support older unpolarized measurements within their larger errors. The discrepancy with one recent experiment is possibly due to a remaining model dependence in the analyses of this type of measurements. In this case the double polarization observables contribute information which is complementary to unpolarized measurements concerning the separation of the non-resonant background contributions.

\section{ACKNOWLEDGEMENTS}

The experiments have been performed within the collaborations A1 and A3 at MAMI with contributions from institutes of the universities of Basel, Bonn, Glasgow, Ljubljana, Mainz, Rutgers and Tübingen. The preliminary CMR-results are based on the doctoral thesis of Th. Pospischil. Calculations have been provided by H. Arenhövel concerning the influence of FSI on the polarization observables in quasielastic $e-D$ scattering, and by $\mathrm{S}$. Kamalov and L. Tiator for the extraction of the CMR from the $\pi^{0}$ experiment. Financial support came from the Deutsche Forschungsgemeinschaft (SFB 201).

\section{REFERENCES}

1. H. Arenhövel, Phys. Lett. B 199, 13 (1987), Z. Phys. A 331, 509 (1988)

2. R.G. Arnold et al., Phys. Rev. C 23, 363 (1981)

3. T.N. Taddeucci et al., Nucl. Instrum. Methods A 241, 448 (1985)

4. M. Ostrick et al., Phys. Rev. Lett. 83, 276 (1999)

5. C. Herberg et al., Eur. Phys. J. A 5, 131 (1999)

6. I. Passchier et al., Phys. Rev. Lett. 82, 4988 (1999)

7. T. Eden et al., Phys. Rev. C 50, R1749 (1994)

8. D. Rohe et al., submitted to Phys. Rev. Lett.

9. J. Becker et al., submitted to Eur. Phys. J.

10. S. Platchkov et al., Nucl. Phys. A 510, 740 (1990)

11. C. Becchi and G. Morpurgo, Phys. Lett. B 17, 352 (1965)

12. R. Beck et al., Phys. Rev. Lett. 78, 606 (1997)

13. G. Blanpied et al., Phys. Rev. Lett. 79, 4337 (1997)

14. H. Schmieden, Eur. Phys. J. A 1, 427 (1998)

15. D. Drechsel and L. Tiator, J. Phys. G: Nucl. Part. Phys. 18, 449 (1992)

16. A.S. Raskin and T.W. Donnelly, Ann. of Phys. 191, 78 (1989)

17. K.I. Blomqvist et al., Nucl. Instrum. Methods A 403, 263 (1998)

18. Th. Pospischil, doctoral thesis, Mainz, in preparation

19. M.W. McNaughton et al., Nucl. Instrum. Methods A 241, 435 (1985)

20. D. Drechsel, O. Hanstein, S.S. Kamalov and L. Tiator, Nucl. Phys. A 645, 145 (1999)

21. R. Siddle et al., Nucl. Phys. B 35, 93 (1971), J.C. Alder et al., Nucl. Phys. B 46, 573 (1972), and K. Bätzner et al., Nucl. Phys. B 76, 1 (1974)

22. R. Gothe in Proceedings of Chiral Dynamics, Mainz (1997), p. 124

23. C. Mertz et al., nucl-ex/9902012

24. F. Kalleicher et al., Z. Phys. A 359, 201 (1997)

25. H. Schmieden et al., approved MAMI proposal A1-3/98 